\title{
Doctrina judicial del principio quid pro quo sobre inmunidades de jurisdicción de organizaciones internacionales en Francia e Italia
}

Fecha de recepción: 25 de enero de 2021

Fecha de aprobación: 20 de agosto de 2021

Doi: https://doi.org/10.12804/revistas.urosario.edu.co/acdi/a.10165

\section{Gloria Ramos-Fuentes* \\ Patricio Masbernat ${ }^{* *}$}

Resumen: el presente artículo aborda aspectos de una nueva doctrina dentro del campo del régimen jurídico de las inmunidades de jurisdicción de las organizaciones internacionales, el cual difiere de otros regímenes existentes en derecho internacional, como las inmunidades de los Estados. Si bien las teorías mayoritarias en esta materia son la absoluta, la relativa y la funcional, actualmente se está abriendo paso

* Universidad Autónoma de Chile. Abogada del Ministerio de Relaciones Exteriores del Gobierno de Chile. Licenciada en derecho (Universidad de Chile), magíster en derecho internacional y magíster en derecho de la Unión Europea (Universidad Complutense de Madrid), doctora en derecho por la Universidad Autónoma de Chile. Correo electrónico: gloramos76@gmail.com oRCID: https://orcid.org/0000-0002-8697-2649 ** Abogado de ejercicio libre, licenciado en derecho (Pontificia Universidad Católica de Chile), magíster en derecho (Universidad de Chile), doctor en derecho (Universidad Complutense de Madrid). Profesor de la Universidad Autónoma de Chile. Correo electrónico: patricio.masbernat@uautonoma.cl oRCID: http://orcid.org/0000-00017137-9474

Para citar este artículo: Ramos-Fuentes, Gloria, \& Patricio Masbernat. Doctrina judicial del principio quid pro quo sobre inmunidades de jurisdicción de organizaciones internacionales en Francia e Italia. Anuario Colombiano de Derecho Internacional (ACDI) 15 (2022), 105-135. https://doi.org/10.12804/revistas. urosario.edu.co/acdi/a.10165 
una nueva doctrina denominada por algunos autores y tribunales como quid pro quo. Esta doctrina se ha generado frente a las limitaciones de las doctrinas existentes para resolver plausiblemente cierto tipo de casos caracterizados por acciones judiciales de particulares en contra de organizaciones internacionales, en los cuales los derechos fundamentales y humanos involucrados han requerido ser ponderados frente a los fundamentos de las inmunidades de dichos entes. Este trabajo, luego de explicar sintéticamente las bases de dicha doctrina, busca exponer las principales decisiones judiciales que han recaído en esta clase de casos en Francia e Italia, para desde ahí obtener algunas conclusiones útiles para este campo jurídico.

Palabras claves: organizaciones internacionales; inmunidad de jurisdicción; resolución de conflictos.

\section{Judicial Doctrine of quid pro quo Principle about Immunities of Jurisditions of International Organizacions in France and Italy}

Abstract: This paper addresses aspects of a new doctrine within the field of jurisdictional immunities of international organizations, which differs from other regimes in international law, such as the immunities of States. Although the majority of theories in this matter are the absolute, the relative, and the functional, a new doctrine is currently making its way, known by some authors and courts as quid pro quo. This doctrine aims to face the limitations of the existing doctrines to plausibly resolve certain types of cases - private lawsuits against international organizations - in which fundamental and human rights require to be weighed against the foundations of immunities. After synthetically explaining the theory's doctrinal bases, this paper seeks to expose the main judicial decisions that have dealt with those cases in France and Italy to obtain some useful conclusions for this legal field.

Keywords: International organizations; jurisdictional immunities; conflict resolution. 
Doutrina judicial do princípio do quid pro quo sobre imunidades de jurisdição de organizações internacionais na França e na Itália

Resumo: este artigo aborda aspectos de uma nova doutrina dentro do campo específico do regime jurídico das imunidades jurisdicionais das organizações internacionais, que difere de outros regimes existentes no Direito Internacional, como as imunidades dos Estados. Embora as teorias majoritárias sobre o assunto sejam o absoluto, o relativo e o funcional, uma nova doutrina está em curso, denominada por alguns autores e tribunais de quid pro quo. Esta doutrina foi gerada em face das limitações das doutrinas existentes para resolver de forma plausível certos tipos de casos caracterizados por ações judiciais de indivíduos contra organizações internacionais, em que os direitos fundamentais e humanos envolvidos devem ser ponderados contra os fundamentos das imunidades das referidas entidades. Este trabalho, depois de explicar sinteticamente as bases desta doutrina, procura expor as principais decisões judiciais que recaíram nesta classe de processos na França e na Italia, e a partir daí obter algumas conclusões úteis para este campo jurídico.

Palavras-chave: organizações internacionais; imunidade de jurisdição; resolução de conflitos.

\section{Introducción general a la doctrina de quid pro quo}

El presente artículo aborda aspectos de una nueva doctrina dentro del campo del régimen jurídico de las inmunidades de jurisdicción de las organizaciones internacionales (OOII), que difiere de otros regímenes existentes en derecho internacional como las inmunidades de los Estados. $\mathrm{Si}$ bien las teorías mayoritarias en esta materia son la absoluta, la relativa y la funcional, ${ }^{1}$ actualmente se está abriendo paso una nueva doctrina denominada por algunos autores y tribunales como quid pro quo. ${ }^{2}$

\footnotetext{
1 Por razones de espacio no podremos dar cuenta de ellas y de las diferencias y aproximaciones con la nueva teoria de quid pro quo, por lo que deberemos dejar esa tarea para otra publicación. Para todos: Tom Ruys, Nicolas Angelet y Luca Ferro, The Cambridge Handbook of Immunities and International Law (Cambridge, Cambridge University Press, 2019, https://doi.org/10.1017/9781108283632).

2 Por ejemplo: Trabajadores c. Empleamos S. A., Secretaría Ejecutiva del Convenio Andrés Bello
} 
Esta doctrina ha sido creada frente a las limitaciones de las doctrinas existentes para resolver plausiblemente cierto tipo de casos caracterizados por acciones judiciales de particulares en contra de dichos entes, en los cuales los derechos fundamentales y humanos involucrados han requerido ser ponderados y evaluados equilibradamente frente a los fundamentos de las inmunidades de las ooli. ${ }^{3}$

La doctrina quid pro quo tiene un contenido complejo - que no podrá ser desarrollado completamente en este espacio-, cuya comprensión varía entre autores y tribunales, pero que en un sentido general podría conceptualizarse como una obligación de las OOII de ofrecer medios alternativos de solución de controversias en los conflictos con particulares del Estado sede o de un Estado miembro, como una condición al reconocimiento de la inmunidad de jurisdicción de las ooII, cuyo objetivo sea determinar una eventual o posible responsabilidad de la organización internacional (OI) y su obligación de reparación por los daños que se deriven de la misma. ${ }^{4}$

(SECAB) y la Organización de Estados Iberoamericanos (OEI), AL3295-2014, Corte Suprema de Justicia, Sala de Casación Laboral (abril 9, 2014), https://www.cortesuprema.gov. co/corte/wp-content/uploads/relatorias/la/Publicacion/AL3295-2014.pdf; William M. Berenson, Inmunidad de jurisdicción de organizaciones internacionales públicas en el sistema interamericano: desarrollos y preocupaciones, (Organización de Estados Americanos, 2013), https://www.oas.org/es/sla/ddi/docs/publicaciones_digital_XL_curso_derecho_ internacional_2013_William_M_Berenson.pdf

3 Rosa Freedman, "UN Immunity or Impunity? A Human Rights Based Challenge," The European Journal of International Law 25 no. 1 (2014): 239-254, https://doi.org/10.1093/ ejil/cht082; Martha Rutsel, «International Financial Institutions and Claims of Private Parties. Immunity Obliges,» The World Bank Legal Review 3 (2011), https://doi. org/10.1596/9780821388631_CH03; Emmanuel Gaillard e Isabelle Pingel-Lenuzza, «International Organisations and Immunity from Jurisdiction: To Restrict or to Bypass,» International \& Comparative Law Quarterly 51 no. 1 (enero, 2002): 1-15, https://doi. org/10.1093/iclq/51.1.1; Jhon Griffth, "Restricting the Immunity of International Organizations in Labor Disputes: Reforming an Obsolete Shibboleth," Virginia Journal of International Law 25 (1985): 1007-ss.; August Reinisch, International Organizations before National Courts (New York: Cambridge University Press, 2008); Rosanne Van Alebeek y Andre Nollkaemper, Privileges and Immunities of International Organizations in the Case Law of Dutch Courts, (Amsterdam Center for International Law no. 2012-11), https:// hdl.handle.net/11245/1.417848

4 Hemos excluido de este estudio la responsabilidad internacional por el hecho ilícito. Laura Huici Sancho, El hecho internacionalmente ilícito de las organizaciones internacionales. El proyecto de la Comisión de Derecho Internacional sobre responsabilidad de las organizaciones internacionales, (Barcelona, J.M. Bosch, 2007). 
La doctrina surge de la necesidad de superar el vacío que dejan las oOII al no ofrecer medios idóneos y efectivos de solución de conflictos $^{5}$ para responder y reparar por sus actos dañosos (accountability). ${ }^{6} \mathrm{El}$ ícono de estos temas son los casos en que se han visto involucradas las Naciones Unidas (ONU); una referencia que en absoluto resuelve toda la complejidad de esta clase de casos. ${ }^{7}$

Ahora, en adelante veremos una serie de sentencias, que no siguen una misma doctrina uniforme (pues el criterio de quid pro quo se encuentra en formación) y que en general constituyen excepciones frente a la regla general de acoger la inmunidad de las OOII. Por ejemplo, Young ${ }^{8}$ comenta $^{2}$ respecto de Austria que los tribunales han otorgado inmunidad absoluta a las OOII, siempre que actúen dentro de sus funciones, en interés por atraerlas a Viena a fin de obtener reputación internacional e influencia en las relaciones internacionales. Añade este autor que, tal vez debido a la inutilidad de presentar una demanda contra ellas en los tribunales austriacos, la mayoría de las disputas se resuelven mediante un procedimiento de mediación, donde el Ministerio de Relaciones Exteriores de Austria actúa como mediador. Agrega también que si bien ello requiere

5 August Reinisch, "To What Extent Can and Should National Courts Fill the Accountability Gap?” International Organizations Law Review 10 (2013): 572-587.

Este autor concluye lo siguiente: "A rule of law-based international environment will continue to put pressure on international organizations to close accountability gaps. The exercise of power, whether on the domestic or the international plane, requires control and possibly review by judicial bodies. Where international organizations are not subject to the jurisdiction of international supervisory or adjudicative bodies and enjoy totally unrestricted immunity, this may create a lack of accountability."

También ver: Karel Wellens, «Fragmentation of International Law and Establishing an Accountability Regime for International Organizations: The Role of the Judiciary in Closing the Gap», Michigan Journal of International Law (2004): 1159-ss.

6 Ved Nanda, «Accountability of International Organizations: Some Observations», Denver Journal of International Law and Policy 33 no. 3 (junio, 2005): 379-390.

7 Nico Schrijver, "Beyond Srebrenica and Haiti: Exploring Alternative Remedies against the United Nations," en Immunity of International Organizations 55, (Holanda, Brill Nijhoff, 2015), 329-341. https://doi.org/10.1163/9789004296060_017

8 Carsonn Young, «The Limits of International Organization Immunity: An Argument for a Restrictive Theory of Immunity Under the IOIA,» Texas Law Review 95 no. 4 (2017): 909-910, https://texaslawreview.org/wp-content/uploads/2017/03/Young.pdf 
la aquiescencia de la OI, esta vía evita el sesgo implícito de los tribunales administrativos de dichas entidades. ${ }^{?}$

\section{Francia ${ }^{10}$}

\subsection{Introducción ${ }^{11}$}

Reinisch sostiene que tradicionalmente los tribunales franceses han desestimado las acciones en contra de las ooil bajo el argumento de que interferirían con la independencia de sus operaciones. Sin embargo, existen algunos litigios que plantean un punto de vista diferente (en donde los tribunales desconocen esta inmunidad si el demandante ha sido desprovisto de un foro para presentar su demanda), que son los que estudiaremos en este apartado. ${ }^{12}$

Para Schmitt, la obligación de la or de otorgar una vía para la solución de controversias no constituye una exigencia de ius cogens, sino un asunto de orden público internacional. ${ }^{13}$ Ello se alinea con la doctrina de los tribunales franceses. Normalmente, en Francia las sentencias relacionadas con OoII son emitidas por la Cámara Social del Tribunal de Casación porque usualmente estas disputas están relacionadas con

9 Kristen Schmalenbach, "Austrian Courts and the Immunity of International Organizations," International Organizations Law Review 10 no. 2 (2013): 461, https://doi. org/10.1163/15723747-01002012

Este autor señala que “The Austrian courts' silence about alternative means of dispute settlement provided by international organizations is conspicuous."

10 Otros casos en Francia: De Beaugrenier c. Unesco (2009), Court of Cassation, 11 February 2009, application $\mathrm{N}^{\circ}$ 07-44240; Mazéas c. Latin Union (2003), Court of Cassation, 30 September 2003, Juris-Classeur Périodique (2004) II 10102; X c. Organisation Européenne de télécommunications par satellite (EUTELSAT) (2001), Court of Cassation, 5 June 2001, Bulletin (2001).

11 Christian Dominice', “Observations sur le contentieux des organisations internationales avec des personnes prive'es", Annuaire Français de Droit International 45 (1999): 623-648. https://www.persee.fr/doc/afdi_0066-3085_1999_num_45_1_3580

12 August Reinisch, "Privileges and Immunities", en The Oxford Handbook of International Organizations, eds. Cogan et al. (Oxford: Oxford University Press, 2016), 1064, https:// doi.org/10.1093/law/9780199672202.003.0049

13 Pierre Schmitt, Access to Justice and International Organizations: The Case of Individual Victims of Human Rights Violations (UK: Edward Elgar Publishing, 2017), 290-293. 
asuntos laborales. En algunos casos, los tribunales sostuvieron la inmunidad absoluta solo por razones convencionales formales. ${ }^{14}$

Schmitt explica que en $2005,{ }^{15}$ el Tribunal de Casación francés cambió su práctica en el caso African Development Bank c. Degboe (2005), ${ }^{16}$ pues dicha organización no había establecido ningún tribunal para decidir sobre tales casos. El Tribunal de Casación observó la imposibilidad de que una persona ejerciera un derecho perteneciente al orden público internacional, lo que constituía una denegación de justicia que fundamentaba la competencia de los tribunales franceses en casos con una conexión francesa, en este caso, la nacionalidad francesa del demandante.

Ahora, para este autor, la postura de los tribunales franceses es meramente formal, de un control in abstracto, sin hacer referencia a la práctica del tribunal al cual se ha otorgado el acceso, que para Schmitt constituiría una presunción de que la OI ha establecido un procedimiento independiente e imparcial y, por lo tanto, corresponde demostrar lo contrario al demandante individual. Solo en ausencia de un mecanismo alternativo de solución de controversias se levanta la inmunidad de la OI. Cuando existen tales tipos de mecanismos, las jurisdicciones francesas concluyen rápidamente que estos mecanismos de solución de controversias ofrecen garantías suficientes. Así fue el análisis en el caso Illemassene c. OCDE (2010) ${ }^{17}$

14 X c. Eutelsat (2001), Court of Cassation (soc.), 5 June 2001, Bulletin (2001)161 (France); Maréas c. Latin Union (2003), Court of Cassation, 30 September 2003, Juris-Classeur Périodique (2004) II 10102 (France).

15 Schmitt, Access to Justice..., 291.

Con todo, debe considerarse el análisis de Kolb respecto de que ambos conceptos y sus relaciones y diferencias: «Toute confusion entre ce qu'on a appelé ordre public international et le ius cogens doit être scrupuleusement évitée. .... L'ordre public n'est pas égal au ius cogens. L'une et l'autre notion sont sur des plans juridiques nettement distincts : l'ordre public est une notion de droit matériel, le ius cogens une technique juridique. L'ordre public est une notion imprégnée de causes spécifiques et de finalités sociales; le ius cogens ne produit qu'un effet juridique technique et c'est à lui que se limite sa définition». Al explicar las teorias sobre ius cogens aborda los criticas de aquellas que lo consideran equivalente al orden público internacional (especialmente, teorías del orden público internacional, ius cogens como regla axiológica de una comunidad, sean restrictivas, extensivas y estatistas).

Robert Kolb, Théorie du Ius Cogens International. Essai de relecture du concept, (Geneve: Graduate Institute Publications, 2001), 59-168 y 172-181.

16 African Development Bank c. Degboe (2005), Court of Cassation (soc.), 25 January 2005, (2005) ILDC 778 FR 2005 (France).

17 Illemassene c. OECD (2010), Court of Cassation (soc.), 29 September 2010, Nº 09-41030, ILDC 1749 (FR 2010) (France). 
y en Beaugrenier c. Unesco (2009). ${ }^{18}$ En este último, explica, el Tribunal de Casación evaluó como adecuado el procedimiento de arbitraje para tratar los asuntos relacionados con el personal que otorgaba la oI, y el tribunal arbitral no tenía la obligación de indicar las razones subyacentes de la decisión, donde el único árbitro fue nombrado previa y exclusivamente por la OI, quien además era simultáneamente el presidente de la Junta de Apelaciones Interna Conjunta, por ello la corte concluyó que ello no era contrario al orden público internacional.

Finalmente, este autor recuerda que en Hintermann c. Western European Union (1995), ${ }^{19}$ la Corte de Casación rechazó la demanda del tercero afectado bajo la justificación de ser ello necesario para mantener las relaciones internacionales con las numerosas OOII de las que Francia era un Estado miembro.

\subsection{Unesco c. Boulois (1997) ${ }^{20}$}

Este litigio versó acerca de un contrato celebrado por la Unesco con el señor Max-Henri Bouloi, el cual contenía una cláusula de arbitraje que el segundo invocó para iniciar el procedimiento. El particular nombró un árbitro, pero la Unesco por su parte se negó a hacerlo. El afectado demandó judicialmente a la OI. El tribunal de primera instancia rechazó la excepción de inmunidad de jurisdicción sosteniendo que la estipulación de una cláusula de arbitraje implicaba una renuncia a la inmunidad. La sentencia fue apelada, y el Tribunal de Apelación de París la confirmó sobre la base de que la inmunidad de jurisdicción de la Unesco no puede permitirle apartarse del principio de pacta sunt servanda al negarse a proceder a la designación de un árbitro de conformidad con la cláusula de compromiso contenida en el contrato con el señor Boulois. Por otro lado, acoger la inmunidad conduciría inevitablemente a que se le impida presentar su caso ante un tribunal, lo que es contrario al orden público en el sentido de que constituye una denegación de justicia y una violación

18 De Beaugrenier c. Unesco (2009), Court of Cassation, 11 February 2009, 4 Journal de Droit International (2009), 1247-1274.

19 Hintermann c.Western European Union (1997), Court of Cassation, 14 November 1995, Journal du Droit International (1997), 141.

20 Unesco c. Boulois (1998), Tribunal de grande instance de Paris, 20 octobre 1997, Rev. Arb. (1997) 575; Cour d'appel Paris (14e Ch. A), 19 juin 1998 
de las disposiciones del artículo 6. 1. del Convenio para la Protección de los Derechos Humanos y de las Libertades Fundamentales (CEDH).

\subsection{X. c. Banque Africaine de Développement (2005) ${ }^{21}$}

En esta causa judicial, la Corte de Casación de Francia acogió una demanda en contra del Banco Africano de Desarrollo a pesar de que el demandante había sido empleado por una OI que operaba en África. La decisión tomó en cuenta la circunstancia de que dicho empleado poseía la nacionalidad francesa (lo que determinó una conexión con Francia), en cuyos términos se encontraba protegido por el CEDH, que también (obviamente) vincula al Estado francés. En este caso, la OI demandada no podía hacer uso de la inmunidad de jurisdicción, ya que en el momento de los hechos no había instituido un tribunal con jurisdicción para resolver disputas de tal naturaleza. Para el tribunal, decidir lo contrario implicaría una denegación de justicia y obstaculizar el ejercicio de un derecho que se enmarca en el orden público internacional de Francia.

\subsection{Beaugrenier c. Unesco (2009) $)^{22}$}

Se trata de una causa laboral respecto de personal del Economat que tenía una vinculación especial con la Unesco. El contrato de trabajo estipulaba expresamente que "sus otras condiciones de empleo y trabajo se regirían por el reglamento del personal del Economat complementado por la legislación laboral francesa". Sin embargo, la Corte de Apelación consideró que un contrato de trabajo no merecía esa renuncia de la inmunidad de jurisdicción. A juicio de la Corte de Casación, esa decisión violaba el Acuerdo de Sede celebrado entre Francia y la Unesco y el Código del Trabajo.

Añadió que el artículo 21 del reglamento de personal de la Unesco define que las disputas con el personal se resolverán por un arbitro unipersonal, cuya decisión será apelable ante una comisión arbitral, lo que a juicio de la Corte de apelación otorgaría las garantías de un proceso justo. Sin embargo, el personal del Economat no es parte del personal de la

21 M.X. c. Banque Africaine de Développement (2005), Cour de Cassation, Chambre sociale, Audience publique du 25 janvier 2005, $\mathrm{N}^{\circ}$ de pourvoi: 04-41012, Publié au bulletin. Bulletin $2005 \mathrm{~V}, \mathrm{~N}^{\circ} 16,13$.

22 De Beaugrenier c. Unesco (2009), Cour de cassation, civile, Chambre sociale, 11 février 2009, 07-44.240, Publié au bulletin, Fecha de Resolución:11 de Febrero de 2009.

ACDI, Bogotá, ISSN: 2027-1131/ISSNe: 2145-4493, Vol. 15, pp. 105-135, 2022 
Unesco, por lo que el demandante no puede acceder a la vía de apelación descrita. Para la Corte de Casación esto violaría el artículo 6. 1. del CEDH y el artículo 12 del Acuerdo de Sede celebrado entre Francia y la Unesco.

No obstante, el procedimiento fijado para el demandante no violaba «la conception française de l'ordre public international» (dado que no implicaba la renuncia de la inmunidad de la Unesco sino solo una regulación del modo de zanjar disputas), por lo que rechazó el recurso (expresó que la mera referencia a la ley francesa en la carta de compromiso del empleado no constituye una renuncia a la inmunidad de jurisdicción de que goza la Unesco sobre la base del artículo 12 del Acuerdo de Sede de 2 de julio de 1954).

Lo relevante de la causa se encuentra en la declaración vertida en el fundamento 2 de su sentencia, en el cual el tribunal sostiene que una OI no puede invocar la inmunidad de jurisdicción sobre un empleado si no ha instituido en ella un tribunal para resolver las disputas que surjan de la relación laboral y una resolución conforme a un procedimiento que ofrezca garantías de un juicio justo en el sentido del artículo 6. 1. del CEDH, lo que incluye los derechos a actuar dentro de un periodo de tiempo suficiente, a ser asistido por un abogado de su elección y a obtener una decisión motivada que se pronuncie sobre la disputa:

\begin{abstract}
$2^{\circ}$. (...) qu'une organisation internationale ne peut se prévaloir de l'immunité de juridiction à l'égard d'un salarié si elle n'a pas institué en son sein un tribunal chargé de trancher les différends découlant de la relation de travail et statuant selon une procédure offrant les garanties d'un procès équitable au sens de l'article $6 \int 1$ de la Convention européenne de sauvegarde des droits de l'homme; qu' au nombre de ces garanties, figurent le droit d'agir dans un délai suffisant, le droit de se faire assister par un conseil de son choix et enfin la motivation de la décision statuant sur le différend.
\end{abstract}

\title{
2.5. Illemassene c. OCDE $(2010)^{23}$
}

Se trató de una demanda laboral en contra de una OI, y si bien el demandante - un funcionario de la Organización para la Cooperación y el Desarrollo Económicos (OCDE) - reconoció que existía un tribunal

23 Illemassene c. OCDE (2010), Cour de cassation, Chambre Sociale, Audience publique du mercredi 29 septembre 2010, $\mathrm{N}^{\circ}$ de pourvoi: 09-41030. Non publié au bulletin. 
administrativo de la OCDE para resolver el litigio, este no era ni imparcial ni independiente, por lo que "se violó la concepción francesa de política pública internacional” y el artículo 6. 1. del CEDH. El tribunal desestimó la demanda porque consideró que la OCDE proveyó de un tribunal administrativo que entregaba garantías suficientes al trabajador de respeto a su derecho de acceso al tribunal y a un proceso equitativo, en términos que no eran contrarios al diseño francés del orden público internacional. ${ }^{24}$

Berenson explica que en este caso, decidido por sentencia (rejet) de la Corte de Casación (Chambre Sociale), el demandante mantuvo que el Tribunal de la OCDE violó sus derechos garantizados por el CEDH de acceder a un debido proceso y un juicio imparcial sobre sus reclamos porque los jueces fueron nombrados por la misma organización, los juicios no fueron públicos y no hubo derecho a apelación. La corte francesa desestimó la demanda con base en la inmunidad y concluyó que los jueces del tribunal de la OCDE podían ejercer sus funciones con plena independencia e imparcialidad y que el mecanismo establecido por dicha entidad para dirimir conflictos con su personal garantizó un debido proceso adecuado. Este jurista norteamericano ha tomado la posición de que los tribunales administrativos establecidos por las OOII no pueden cumplir con los estándares modernos del debido proceso porque, en su opinión, a los jueces elegidos por la misma organización les faltaría la independencia requerida. Este defecto, sostiene, solo puede ser rectificado con la participación de los funcionarios en el proceso de selección a fin de equilibrar los intereses de la OI y del funcionario. ${ }^{25}$

Berenson explica:

la Corte de Casación hizo una evaluación de los procedimientos del Tribunal de la organización, incluyendo el nombramiento de sus jueces. Concluyó que el nivel de justicia ofrecida por el Tribunal fue ‘adecuado' y, por lo tanto, el reconocimiento de inmunidad de la

24 Drago sostiene que «Le droit international est à la recherche d'un e'quilibre entre les exigences d'accueil des lois ainsi que des situations juridiques e'trangères et la cobe'rence de l'ordre juridique français. L'ordre public est le garant de cette inte'grite', selon l'expression du professeur Le'na Gannage" ».

Guillaume Drago, "Conception française de l'ordre public international", Le Rapport Annuel 2013. https://www.courdecassation.fr/files/files/Publications/Rapport\%20 annuel/rapport-annuel_2013.pdf

25 Berenson, Inmunidad de jurisdicción de organizaciones internacionales... 
organización en los tribunales franceses con respecto a la demanda del funcionario no violó la obligación de estado de garantizar el derecho humano de debido proceso y adjudicación imparcial de sus derechos. ${ }^{26}$

\section{Italia}

\subsection{Introducción}

Young ${ }^{27}$ considera que las aproximaciones de los tribunales de Italia, Inglaterra y Austria son mejores que las de los tribunales de EE. UU., y que estos últimos podrían incorporarlas a su jurisprudencia. Este autor explica que los tribunales italianos han interpretado restrictivamente las normas sobre inmunidad de jurisdicción, diferenciando entre acta iure gestionis y acta iure imperii, reconociendo la inmunidad solo respecto de estas últimas. Cita el caso Instituto Nazionale di Previdenze per i Dirigenti di Aziende Industriali (INPDAI) c. FAO (1982), ${ }^{28}$ que trata de una demanda de un propietario contra la FAO por no pagar la renta de uno de los edificios que ocupaba y el aumento del monto de acuerdo al contrato. El acuerdo entre la FAO e Italia (1950) otorga inmunidad de jurisdicción a la organización salvo su propia renuncia expresa. El tribunal de casación italiano negó inmunidad de jurisdicción a la FAO sosteniendo que siempre que las OOII actuaban en el campo del derecho privado (derecho comercial, alquiler de inmueble para usos diferentes a sus funciones), se colocaban en la misma posición que las personas privadas con quienes

\footnotetext{
26 Ibíd, 234.

Véase también: Griffth, "Restricting the Immunity of International Organizations in Labor Disputes...,» 1007-ss.

27 Young, «The Limits of International Organization Immunity...».

28 Corte di cassazione; Sezioni unite civili; sentenza 18 ottobre 1982, No 5399, INPDAI c. FAO, Rivista di giurisprudenza costituzionale e civile, Il Foro Italiano, V. 105. Parte Prima: Giurisprudenza Costituzionale e Civile (1982), 2977.

La disputa se relacionaba con una propiedad arrendada, que sin embargo no se usaba como sede, sino que se usaba para fines privados, por lo tanto, aplicando el principio de inmunidad restringida, no había una conexión directa y necesaria de la propiedad con los propósitos institucionales de la autoridad. La sentencia sostuvo que: "[in] considerazione del carattere privatistico del contratto di locazione concluso nella specie con la FAO e della impossibilità di ricondurre tra gli scopi primari della medesima la scelta del luogo dove sistemare gli uffici".
} 
habían firmado contratos y, por lo tanto, renunciaban al derecho de actuar como organismos soberanos — distinción entre actos iure gestionis y iure imperii, no reconociendo inmunidad absoluta a la $\mathrm{FAO}$ - Para reconocer la inmunidad, durante mucho tiempo, los tribunales italianos buscaban una relación directa entre la actividad efectuada y los fines de la organización.

En juicios más recientes, el tribunal de casación se ha inclinado por extender la inmunidad de la FAO (sosteniendo que el Acuerdo de la Sede de Italia con dichsa OI impide que se presenten juicios contra ella en los tribunales italianos). Por ejemplo: en Colagrossi c. FAO (1992), ${ }^{29}$ se estableció que la inmunidad de jurisdicción se extendía no solo a los activos de la organización sino también a la propia institución, por lo que alcanza a sus disputas laborales; en G.C. c. FAO (1999), ${ }^{30}$ el tribunal ratificó la inmunidad de la organización por una disputa laboral con un empleado italiano; en Carreti c. FAO (2004), ${ }^{31}$ el decisor afirmó no solo la inmunidad de la jurisdicción italiana, sino también la competencia del Tribunal Administrativo de la Organización Internacional del Trabajo (ОIT) para la resolución de controversias entre la FAO y sus empleados. ${ }^{32}$

Para Berenson, el cambio de opinión se debe a una amenaza de la FAO de llevar la cuestión a la Corte Internacional de Justicia (CIJ) para una opinión consultiva, alegando incumplimiento con la obligación de reconocer la inmunidad absoluta consagrada por la Convención de la ONU sobre las Prerrogativas e Inmunidades de los Organismos Especializados. Posteriormente, el Gobierno italiano y la FAO concertaron

29 FAO c. Colagrossi (1992), Court of Cassation s.u., 18 May 1992, N 5942, RDIPP $1993,400$.

30 G.C. c. FAO (1999), Corte de Casación, sentencia del 5 de noviembre de 1999.

31 Caretti c. FAO (2004), Court of Cassation s.u., 23 January 2004, $\mathrm{N}^{\circ}$ 1237, AC, 2004, 1328.

32 Sara Consolino, L'immunità delle organizzazioni internazionali: il caso della FAO, Corso Di Laurea Magistrale In Relazioni Internazionali, Libera Università Internazionale degli Studi Sociali, 2017, 94.

Consolino concluye que de las diversas sentencias dictadas a lo largo del tiempo por los tribunales italianos en los casos relacionados con la FAO, se puede decir que, aparte de las disputas relacionadas con el trabajo con sus empleados, nunca se reconoce una inmunidad absoluta a la organización, sino una "inmunidad limitada", que distingue entre actividades de carácter público, necesarias para desempeñar las funciones de la entidad y, por lo tanto, exentas de la jurisdicción italiana, y actividades de carácter privado. 
y firmaron un acuerdo de sede en el cual reconocieron la inmunidad absoluta de la FAO, a cambio del establecimiento por parte de esta OI de mecanismos adecuados para la resolución de conflictos con terceros que surjan en derecho privado. ${ }^{33}$

En efecto, Reinisch explica que en diversos casos los tribunales italianos han confirmado la inmunidad de las OoII en disputas laborales siempre que hayan establecido procedimientos alternativos efectivos de solución de controversias, sobre el respaldo de la jurisprudencia del caso Waite \& Kennedy c. Germany (TEDH, 1999). ${ }^{34}$

Novak y Reinisch ${ }^{35}$ recuerdan el caso Pistelli c. European University Institute (EUI) (2005), ${ }^{36}$ en el cual el tribunal de apelación italiano aceptó la inmunidad de la OI respectiva siempre y cuando garantizase la protección jurisdiccional en situaciones similares ante un juez imparcial e independiente, incluso si se basaba en procedimientos y criterios diferentes de los que se encuentran en el ordenamiento jurídico interno. ${ }^{37}$ El tribunal sostuvo que en tales casos no se habían violado los "principios cardinales" de la Constitución italiana y que no había razón para no aplicar la convención respectiva que otorga inmunidad. El tribunal de apelación determinó que la EUI había establecido un cuerpo para resolver disputas que era un órgano verdaderamente judicial en lugar de un órgano interno de toma de decisiones. Para el tribunal de apelación, explican los autores, esto podría deducirse del hecho de que la selección de los

33 Berenson, Inmunidad de jurisdicción de organizaciones internacionales...

Este autor cita a August Reinisch, International Organizations Before National Courts, 131-132.

34 Reinisch, "Privileges and Immunities", 1066.

35 Gregor Novaky August Reinisch, "Issues of Effectiveness and Legitimacy. Desirable Standards for the Design of Administrative Tribunals from the Perspective of Domestic Courts," The Development and Effectiveness of International Administrative Law, ed. Olufemi Elias (Leiden, Martinus Nijhoff Publishers, 2012), 286.

36 Pistelli c. European University Institute (2005), Appeal judgment, No 20995; ILDC 297 (IT 2005), Guida al diritto 40 (3/2006) (in Italian), 28 October 2005.

37 Novak y Reinisch, "Issues of Effectiveness and Legitimacy...", 286.

"ILDC translation, at 14.1 ("However, the situation is different for a convention which only excludes such disputes from Italian courts, while nevertheless ensuring the jurisdictional protection of the same situations before an impartial and independent judge, even if chosen with procedures and criteria other than those in national legislation. In this case there is no violation of the "cardinal principles" of our Constitution and no reason not to apply the convention, in the form of the ratifying law")." 
miembros del comité se hacía a partir de una lista compilada por un órgano judicial internacional satisfacía los requisitos de independencia e imparcialidad. ${ }^{38}$ Este comité fue considerado como equivalente a un tribunal ("an alternative or optional remedy to State justice" ... "the body for settling disputes is a truly jurisdictional body”). Además, el Tribunal de Apelaciones señaló que, dado que la UEI había sido creada por los países miembros de la Unión Europea para promover la importancia del patrimonio cultural europeo, sus tradiciones constitucionales y sus instituciones, no podía basarse en una convención que fuera contraria a los valores cardinales de la "institucionalidad europea y su ius cogens".

Novak y Reinisch, además, comentan que la sentencia del Tribunal de Casación italiano en el caso Drago c. Instituto Internacional de Recursos Fitogenéticos (IPGRI) $(2007)^{39}$ se fundamenta en las sentencias de Colagrossi c. FAO (1992), ${ }^{40}$ Carettic. FAO (2004) y Pistelli c. EUI (2005). En ella, exponen, para el Tribunal era constitucionalmente legítima la inmunidad otorgada a una oI cuando la convención que eximía ciertos conflictos de la jurisdicción italiana garantizaba la protección judicial de esos litigios ante un juez imparcial e independiente aunque elegido de acuerdo con procedimientos y criterios diferentes de los que se encuentran en el ordenamiento jurídico interno. Ahora, el tribunal agregó que en el momento pertinente no había posibilidad de apelar al ILOAT (Administrative Tribunal of the International Labour Organization) debido a la jurisdicción restringida ratione temporis de este último. Al mismo tiempo, las reglas internas de la organización establecían que las medidas disciplinarias debían ser reexaminadas por un organismo conocido como el Comité de Apelaciones, que también debía considerar aquellas de naturaleza no disciplinaria.

Sin embargo, esto simplemente constituía un mecanismo interno que no brindaba protección judicial bajo el estándar requerido. Específicamente, las reglas internas excluyeron expresamente la posibilidad de que ese organismo examinara las apelaciones relacionadas con la

38 Sentencia, traducción de Novak y Reinisch, "Issues of Effectiveness and Legitimacy...", 286, nota 53.

39 Drago c. International Plant Genetic Resources Institute (IPGRI) (2007), Final appeal judgment, No 3718 (Court of Cassation, All Civil Sections); ILDC 827 (IT 2007); Giustizia Civile Massimario, 2007, 2, 19 February 2007.

40 FAO c. Colagrossi, 18 de Mayo de 1992, Corte di cassazione, N $^{\circ}$ 5942, 75, Rivista di diritto internazionale (1992) p. 407. 
expiración del contrato de trabajo de un empleado. En consecuencia, terminan estos autores, un empleado posiblemente no tenía acceso a la protección judicial ante un órgano independiente. Esta exclusión de cualquier forma de protección judicial de los empleados de la organización llevó al tribunal a concluir que el IPGRI (International Plant Genetic Resources Institute) no podía confiar en su inmunidad y que la disputa respectiva estaba dentro de la jurisdicción de los tribunales italianos (en el caso Drago c. Instituto Internacional de Recursos Fitogenéticos, 2007). ${ }^{41}$

Para Bonafe, las cortes italianas han sido pioneras en reconocer que la inmunidad de jurisdicción de las OOII debe estar subordinada a la disponibilidad de vías alternativas de solución de conflictos (remedies), ${ }^{42}$ que se fundamenta en el derecho de acceso a la jurisdicción. Este argumento ha tratado de ser rebatido alegando que el orden interno y el orden internacional son diferentes, y que las garantías constitucionales no se aplican a este último, lo que ha sido progresivamente desechado por la Corte Suprema italiana. Bonafe añade que decisiones judiciales más recientes tienden a distinguir con más claridad entre la inmunidad del Estado y de las OOII, y respecto de estas últimas exigen las vías alternativas de solución de conflictos como condición para el otorgamiento de la inmunidad de jurisdicción. La inmunidad de los Estados proviene del derecho de la costumbre internacional; además, respecto de los otros Estados, el tercero afectado siempre puede acudir a sus tribunales internos. Al contrario, en el sistema jurídico italiano la inmunidad de las OOII es otorgada de acuerdo al Derecho de los Tratados y subordinada a la Constitución italiana, por lo que los tratados no pueden derogar

41 Novak y Reinisch, "Issues of Effectiveness and Legitimacy...”, 288.

42 Beatrice Bonafe, "Italian Courts and the Immunity of International Organizations," International Organizations Law Review 10 (2014): 520.

Cita el caso del siguiente modo: Tribunale Di Santa Maria Capua Vetere, Luggeri c. ICEM, Judgment of 20 June 1966, Rivista Di diritto internazionale, 1968”. Asimismo, cita otras sentencias: "Corte di Cassazione, Maida c. Amministrazione per gli aiuti internazionali, judgment of 27 May 1955, Rivista di diritto internazionale, 1956, 548 Tribunale di Bari, ICAMAS c. Scivetti, judgment of 23 December 1975, Rivista di Diritto internazionale, 1976, 574; Corte di Cassazione, Iasbez c. ICAMAS. Judgment No 4502/1977, Rivista di Diritto internazionale, 1978, 579; Tribunale di Roma, Cristiani c. IL AI, Judgment of 17 September 1981, Rivista di diritto internazionale, 1984, 670-671”. 
las normas constitucionales ni el derecho constitucional de acceso a la jurisdicción. ${ }^{43}$

Bonafe agrega que los tribunales italianos han ponderado dos intereses contrapuestos, la inmunidad de jurisdicción de las OOII y el derecho de acceso a la jurisdicción ${ }^{44}$ como en el caso de la Corte Suprema al acceder a la inmunidad de la FAO por otorgar acceso a través de los tribunales de la ОІт: ${ }^{45}$

A positive outcome of the balancing process - together with the grant of immunity - would be secured when the treaty establishing the international organization or the headquarters agreement includes a provision on the duty of the international organization to provide alternative judicial remedies, and effective remedies are actually established for the benefit of international organizations' employees. ${ }^{46}$

En algunos casos en que no se otorgaron vías alternativas de solución de conflictos, se ha rechazado la excepción de inmunidad de jurisdicción. ${ }^{47}$ En otras sentencias, la Corte Suprema italiana se ha mostrado preocupada por asuntos más sustanciales que el solo acceso a una vía de solución de conflictos como la composición del tribunal, su

43 Citados por Bonafe: “Corte Di Cassazione, Pistelli c. EuI, Judgment No 20995/2005”, Rivista di diritto internazionale, 2006, 254 ("la tutela giurisdizionale [...] cede di fronte al principio consuetudinario par in parem non habet iurisdictio, riferito agli Stati [...] Ma una tale prevalenza non ha più giustificazione quando il sacrificio del 'principio cardine' della Costituzione [la tutela giurisdizionale] discende, non già dal fondamento dello stesso ordine internazionale, ma da un impegno liberamente assunto dalla nostra Repubblica attraverso la sottoscrizione di una convenzione"), and Drago c. IPGRI, Judgment $\mathrm{N}^{\circ} 3718 / 2007$, ILDC 827 (IT 2007), para. 6.5."

44 Bonafe, "Italian Courts and the Immunity...», 527 y 521, nota 87.

Cita casos: "Corte Di Cassazione, Inpdai. c. FAO, judgment N ${ }^{\circ}$ 5399/1982, Rivista di diritto internazionale, 1983, 189, Corte di Cassazione, Nacci c. ICAMAS, judgment $\mathrm{N}^{\circ}$ 5565/1994, Rivista di diritto internacional Privato e processuale, 1995, 414".

45 Bonafe, "Italian Courts and the Immunity...", 521, nota 88. Este autor cita casos, Corte Di Cassazione, FAO c. Colagrossi, judgment No 5942/1992, Rivista di diritto internazionale, 1992, 411; y en Corte Di Cassazione, Pistelli c. EUI, Judgment No 20995/2005, Rivista di diritto internazionale, 2006, 254 y Corte Di Cassazione, Nacci c. ICAMAS, judgment $N^{\circ} 5565 / 1994$, Rivista di diritto internazionale privato e processuale, 1995, 415.

46 Ibíd., 274.

47 Ibíd., 522, nota 89. 
imparcialidad e independencia, ${ }^{48} \mathrm{o}$ la disponibilidad y efectividad de vías alternativas de solución de conflictos. ${ }^{49}$ Reinisch también pone de manifiesto esta diversidad de soluciones de las cortes italianas (la exigencia de la vía alternativa de solución de conflictos, la aceptación formal de ellas o la perspectiva más crítica de escrutar acerca de si son efectivas y adecuadas, como en el caso European University Institute IUE c. Piette, 1999, Corte de Casación). ${ }^{50}$ Para Bonafe, una de las principales preocupaciones de la Corte Suprema italiana en las sentencias que analiza es el enfoque de la interacción entre la inmunidad de jurisdicción y el derecho de acceso al tribunal. Sin embargo, el análisis ha sido desde la perspectiva constitucional y no desde el derecho internacional, por ejemplo, desde la jurisprudencia del Tribunal Europeo de Derechos Humanos (TEDH), no obstante ser coincidente con ella. ${ }^{51}$ Esta aproximación es diferente en el caso Drago c. IPGRI (2007), en que previo al análisis constitucional, se razonó que debía efectuarse un análisis de derecho internacional. Bonafe sostiene que la Constitución italiana incorpora el tratado internacional en el ordenamiento jurídico nacional mediante un proceso que incluye una ley del Parlamento. Si existiera un conflicto normativo entre la Constitución y el tratado, el Tribunal Supremo no tendría otra alternativa que hacer una remisión a la Corte Constitucional y solicitar la declaración de nulidad de la legislación nacional que incorpora el tratado con todas las consecuencias que ello implicaría a nivel internacional. Pero tal remisión puede evitarse si la Corte Suprema, a modo de interpretación, logra evitar que surja el conflicto y concilia el derecho a la protección judicial y la norma sobre la inmunidad de las OOII. ${ }^{52}$

De este modo, en Drago c. IPGRI (2007), la Corte Suprema estableció que, desde el derecho internacional, el tratado de sede de la or establecía dos normas que debían interpretarse sistemáticamente; además, al no

\footnotetext{
48 Ibíd., nota 90.

49 Ibíd., nota 91.

50 August Reinisch, "Comments on a Decade of Italian Case Law on the Jurisdictional Immunity of International Organizations," The Italian Yearbook of International Law 19 no. 1 (2009): 105.

51 Bonafe, "Italian Courts and the Immunity...", 518.

52 Beatrice Bonafe, "Italian Courts and the Immunity of International Organizations", Immunity of International Organizations, eds. Niels M. Blokker y Nico J. Schrijve, (Lieden: Brill Nijhoff, 2015), 274.
} 
haber cumplido la obligación de establecer vías alternativas de solución de conflictos quedaba suspendida la inmunidad de jurisdicción. Dicha aproximación de la Corte Suprema podría ser inadecuada para Bonafe por dos razones. Por una parte, si bien parece seguir el caso de la Corte Suprema de Holanda Mothers of Srebrenica Association et al. c. The State of the Netherlands (2012), ${ }^{53}$ este caso no se resolvió de ese modo, y el TEDH apoyó la tesis:

despite the effort to balance the individual right of access to court with the restrictions ensuing from the legitimate aim of affording immunity to international organizations, the ECtHR has accepted that: "It does not follow, however, that in the absence of an alternative remedy the recognition of immunity is ipso facto constitutive of a violation of the right of access to a court" (...) As regards international organisations, this Court's judgments in Waite \& Kennedy and Beer \& Regan cannot be interpreted in such absolute terms either. ${ }^{54}$

Como hemos mostrado, no obstante la posicion zigzageante del TEDH, Reinisch considera que en los tribunales de diversos países, especialmente de Europa, se ha diseminado la tesis más estricta de Waite \& Kennedy c. Germany.

En segundo término, la Corte Suprema italiana no se ha pronunciado en los casos en que los tratados no se refieren a la obligación de una OI de establecer unas vías alternativas de solución de conflictos, lo que Bonafe interpreta como una ausencia de deber preciso en tal sentido. Si los tribunales consideran que todo litigio debe tener un tribunal, esto generaría un conflicto entre tratado y Constitución como ha sido descrito. Otra alternativa es que la Corte Suprema no considere que la ausencia de vías alternativas de solución de conflictos no genere ipso facto una violación a la norma constitucional que consagra el derecho a la jurisdicción (como se ha sostenido respecto del artículo 6 del CEDH), y en tal caso la Corte Suprema debiera aceptar que puede haber disputas sin tribunal: "The only alternative would be to accept that international

53 Dutch Supreme Court, Mothers of Srebrenica Association et al. c. The State of the Netherlands, judgment of 13 April 2012, LJN BW1999.

54 Dutch Supreme Court, Mothers of Srebrenica Association et al. c. The State of the Netherlands, judgment of 13 April 2012, LJN BW1999, para. 164. 
organization immunity is governed, at least in part, by general international law". Para esta autora, existe una clara evolución de la visión de los tribunales italianos en materia de inmunidad de las OOII, su enfoque es claramente restrictivo y cada vez más limitado, particularmente evidente cuando los derechos individuales están en juego, en relación con las disputas laborales y la protección del derecho individual de acceso a los tribunales, y efectúan una importante aplicación de los criterios caso por caso, mostrando un esfuerzo por fundamentar el principio de necesidad funcional e identificar los contenidos centrales de los propósitos públicos esenciales al tiempo que niegan la inmunidad con respecto a las reclamaciones que no implicarían una interferencia indebida con las actividades de la OI. ${ }^{55}$

\subsection{Pistelli c. EUI (Instituto Universitario Europeo) (2005) $)^{56}$}

Esta causa trata sobre una demanda laboral en contra de la EUI, que cuenta con inmunidad de jurisdicción por un tratado con Italia. Esta inmunidad estaría en contraste con el artículo 24 de la Constitución italiana (derecho de acceso al tribunal), tal como está previsto el recurso a una Comisión específica y la eventual intervención del Tribunal de Justicia de la Union Europea. Asimismo se sustenta en la sentencia de casación del caso Pietti c. IUE (1999), ${ }^{57}$ donde se sostuvo que no se otorgaba la inmunidad de jurisdicción a la IUE y que no se le aplicaba la regla de derecho consuetudinario internacional de inmunidad, por lo que debía someterse al tribunal italiano.

El Tribunal de Florencia declaró no compartir los argumentos que apoyaban la decisión adoptada por el Tribunal de Casación de 1999, declarando que el juez nacional no tiene jurisdicción en relación con un conflicto laboral promovido por un empleado del mismo instituto. Añadió que la inmunidad de la jurisdicción italiana de la IUE existiría porque la norma consuetudinaria par in parem non habet iurisdictionem

\footnotetext{
55 Bonafe, "Italian Courts and the Immunity...", (2015), 537.

56 Rivista di Diritto Internazionale Privato e Processuale, Anno XLII, $\mathrm{N}^{\circ} 2$, aprile-giugno 2006, Edizioni Cedam-Padova, pp. 468-474, Corte di Cassazione (s.u.), sentenza 28 ottobre $2005 \mathrm{~N}^{\circ} 20995$

57 IUE c. Piette, Corte di Cassazione, Judgment No149/1999, (18 de marzo de 1999), Rivista di diritto internazionale privato e processuale (2000), 474 y ss.
} 
también se aplica a las OOII, de acuerdo con numerosas decisiones del mismo Tribunal de Casación y, en este caso, una relación de trabajo estaba dentro de los objetivos institucionales de la IUE. Además. la convención constitucional del instituto y el protocolo adjunto, así como el acuerdo posterior de la sede, atribuyeron la inmunidad de la jurisdicción del Estado anfitrión, contemplando los privilegios e inmunidades necesarios para la misión, atribuyendo a los estatutos el poder de definir la disciplina del personal y la constitución de una Junta de Apelaciones como el único órgano designado para resolver los conflictos laborales, con garantías adecuadas de independencia; sobre todo, se estableció la inmunidad frente a ejecuciones e inmunidad respecto de la jurisdicción.

La Corte de Casacion comenta la tradicional doctrina italiana de la extensión de la inmunidad del Estado, regida por regla de derecho consuetudinaria, a las OOII. Esta postura es diferente de la de tribunales de otros países, que consideran que rige una regla convencional (considerato in diritto 4). La sentencia añade que esta problemática es mayor por el surgimiento de sujetos de derecho internacional de características diversas, a los que debe reconocérseles subjetividad internacional, inmunidades y privilegios del mismo modo que a los Estados, de acuerdo al derecho de la costumbre internacional, por lo que parece ser necesario en dicho caso acudir a la fuente convencional (Considerato 9).

Luego, señala que la norma consuetudinaria puede renunciarse por voluntad de la misma or que tiene derecho a la inmunidad, mediante la celebración de convenios con los que se somete sin limitación, generalmente en un ámbito determinado, a la legislación italiana. En la mayoría de los casos, esta separación se efectúa distinguiendo, en los convenios, entre funcionarios con estatuto internacional y funcionarios con estatuto local (Considerato 7). Con todo, existen dudas respecto del grado al que se extiende el principio de paridad de los Estados a las OI (principio par in parem non habet iurisdictionem), por lo que debiera ser reconducido el asunto al artículo 11 de la Constitución italiana y recurrir a los acuerdos de sede con el Estado (Considerato 9).

El Tribunal de Casación argumenta que no existe fundamento del recurso ni aún desde la inmunidad restringida, más aún dado que las tareas realizadas por Pistelli no son puramente patrimoniales. Por ello, se declara que los tribunales italianos no tienen jurisdicción (especialmente por las argumentaciones efectuadas en el Considerato 6, en que distingue actos iure imperii de iure gestionis). La jurisprudencia se ha orientado a sostener que en materia de relaciones laborales, esta solo tiene jurisdicción 
sobre asuntos privados. Para otras relaciones, el mismo juez carece de poder jurisdiccional capaz de interferir en la estructura organizacional y las funciones de las entidades. Precisó además que entre las medidas de naturaleza exclusivamente patrimonial no podrá entenderse la sentencia de condena a un pago que deba ir lógicamente precedida de una comprobación del perjuicio por interrupción de una relación laboral de duración indeterminada, con prestaciones laborales relacionadas con los fines institucionales del organismo empleador. Recuerda que el tratado constitutivo establece ciertas obligaciones de los Estados miembros en relación con el EUI, pero las inmunidades están contenidas en el acuerdo de sede. Dicho acuerdo establece los procedimientoss de solución de controversias con su personal.

La sentencia decide que el EUI goza de inmunidad de jurisdicción (Considerato 13), y que el asunto discutido no es meramente patrimonial. Pero luego, para verificar la procedencia de la inmunidad, la condiciona al respeto del derecho constitucional de tutela judicial efectiva, principio cardinal del ordenamiento italiano (Considerato 14), y a una ponderación entre ambos valores. Respecto de la vulneración de la tutela judicial, lo rechaza al estimar suficiente y adecuada la vía establecida por la oi para resolver los conflictos con el personal, al calificarlo como un medio similar a la justicia italiana, además de considerar que el EUI se formó sobre las bases juridicas comunes europeas.

\subsection{Drago c. IPGRI (Istituto Internazionale per le Risorse Fitogeneriche) (2007)}

El Tribunal de Roma declinó su competencia respecto de la controversia laboral sobre la base de la inmunidad otorgada al IPGRI por el artículo 5 del Acuerdo de sede entre la República Italiana y dicha OI (firmado el 10 de octubre de 1991 y ratificado por ley de 1994). La decisión fue confirmada por el Tribunal de Apelación de Roma (sentencia de 30 de agosto de 2004, N $^{\circ} 3516 / 04$ ), en la que se afirmó que la norma internacional consuetudinaria sobre la inmunidad de Estados extranjeros también era aplicable a las OOII. En este caso, la corte acogió el recurso de casación y

58 Drago c. IPGRI, Cassazione civile, Sez. Un., 19 febbraio 2007, No 3718, Corte Suprema Di Cassazione. Sezioni Unite Civili. 
declaró la jurisdicción de los tribunales italianos, anulando la sentencia impugnada del Tribunale di Roma.

Drago interpuso demanda laboral contra el IPGRI (OI de recursos fitogenéticos), solicitando la nulidad de su despido, su reincorporación e indemnización por daños y perjuicios. Los tribunales de primera y segunda instancia declararon la inmunidad de jurisdicción motivados por la referencia a la norma consuetudinaria que asimila las OOII a los Estados extranjeros para la inmunidad de la jurisdicción, y luego con la afirmación de la falta de jurisdicción del juez italiano sobre la controversia relativa a la determinación de la ilegitimidad del despido con el fin de restablecer la relación. Contra esta decisión, Drago recurrió en casación por violación del derecho constitucional de acceso a la jurisdicción e inadecuada aplicación del derecho internacional relativo a la inmunidad de jurisdicción.

La recurrente sostuvo que durante todo el periodo de la relación laboral y en el momento de su terminación, el IPGRI no se ha unido a ninguna OI para la solución de conflictos laborales con sus empleados ni ha establecido formas adecuadas de apelación de las medidas adoptadas por el Director General. En seguida, la interpretación del tribunal debe estar sujeta a la Constitución, porque se ha violado el derecho a la jurisdicción reconocido en el CEDH (artículos 6 y 13) y la Carta de Derechos Fundamentales de la Unión Europea (artículo 47). Asimismo, se denunció la violación y la aplicación errónea de la norma consuetudinaria de la inmunidad jurisdiccional restringida, argumentando que, en cualquier caso, la jurisdicción del juez italiano sigue teniendo en cuenta la naturaleza de las tareas realizadas por el empleado que no implicaba ninguna participación funcional en la actividad pública de la institución ni la limitación del objeto de la demanda.

La Corte de Casación estudió conjuntamente los puntos puestos en su conocimiento, a través de la cuestión de la inmunidad de jurisdicción. Ella sostuvo que, de acuerdo con el artículo 10 constitucional, el sistema legal italiano se ajusta a las normas de derecho internacional generalmente reconocidas y que de esa norma su jurisprudencia deriva la inmunidad de Estados extranjeros de acuerdo con una costumbre internacional dirigida a respetar la soberanía de los demás Estados. Esta inmunidad concierne a las relaciones jurídicas que son ajenas al sistema legal italiano porque los Estados extranjeros actúan como sujetos de derecho internacional o porque actúan como titulares de un poder de imperio en el orden jurídico. La inmunidad consuetudinaria se extiende 
a los otros sujetos que tienen, en un sentido amplio, la calidad de los órganos del Estado extranjero, incluyendo, en particular, instituciones e institutos de naturaleza cultural (Considerato 6.1.).

Los límites de la inmunidad están marcados por la no trazabilidad de los actos a los poderes soberanos, es decir, entre actos de imperio y actos de gestión. Con respecto a esta distinción y con referencia específica a las relaciones de trabajo, después de algunas incertidumbres, la jurisprudencia de estas secciones unidas se ha orientado en el sentido de que, frente a las entidades ajenas al ordenamiento italiano por ser entidades de derecho internacional e inmunes a la jurisdicción, el juez italiano es titular de la potestad jurisdiccional para todos los litigios relativos a relaciones de trabajo que resulten totalmente ajenas a las funciones institucionales y a la organización de la OI, es decir, constituidos en el ejercicio de capacidades de derecho privado y puede dictar medidas de contenido exclusivamente patrimonial.

Por otro lado, no es seguro que a las OOII se les aplique el principio de derecho de la costumbre internacional "par in parem non habet iurisdictionem", que opera entre los Estados y se refiere el artículo 10 constitucional. Por ello, los privilegios e inmunidades que se les deben reconocer a dichas organizaciones pueden derivar solo de fuentes escritas específicas y por medio del artículo 11 constitucional, que son las convenciones que conforman la organización y los acuerdos de sede. El acuerdo de sede del IPGRI (Roma, 10 de octubre de 1991) otorga inmunidad legal de cualquier tipo, con referencia a cualquier acto de naturaleza pública o privada, excepto en aquellos casos especiales en los que el Director General lo haya renunciado expresamente (Considerato 6.3.).

Desde el Considerato 6.5. en adelante, la Corte de Casación se dedica a analizar la conformidad de esta disposición que reconoce la inmunidad de jurisdicción a las disputas relacionadas con las relaciones laborales con sus empleados a la luz de la garantía constitucional de protección judicial, según el artículo 24 constitucional, que es un principio fundamental del sistema legal italiano. Ahora, este principio cardinal cede al principio consuetudinario "par in parem non habet iurisdictionem", refiriéndose a los Estados, ya que el principio refleja la soberanía igualitaria de estos, que es un fundamento universalmente aceptado por la comunidad internacional, reconocido en el artículo 10 constitucional. Pero tal prevalencia no tiene justificación cuando el sacrificio del principio cardinal de la Constitución se deriva no de la base del propio orden internacional, sino de un compromiso asumido libremente por Italia a 
través de la firma de una convención. En este caso, es necesario que el compromiso asumido se traduzca en una ley de ratificación que obligue a los jueces a poner de relieve los principios fundamentales del orden constitucional con los cuales el compromiso debe ser compatible, bajo la pena de nulidad de la ley de ratificación, como lo ha sostenido la Corte Constitucional. En consecuencia, la exclusión en la esencia del derecho de las personas a la protección judicial ante un órgano independiente e imparcial de las situaciones jurídicas nacientes en un determinado campo de las relaciones debe inducir a dudar de la legitimidad constitucional de la ley de ratificación de convenciones con previsiones similares, es decir, donde sea posible lograr resultados interpretativos de orientación constitucional. Al igual que esos criterios, la jurisprudencia de la Corte de Casación ha sostenido que la inmunidad otorgada a una OI no plantea dudas de legitimidad constitucional cuando la convención que resta esas situaciones del conocimiento del juez italiano garantiza la protección judicial de la misma ante un tribunal imparcial e independiente, aunque elegido con procedimientos y criterios diferentes de los vigentes en la legislación nacional, como en los casos contra la FAO y el Instituto Universitario Europeo (Considerato 6.5.).

Con respecto a la relación laboral en cuestión, se destaca que ella no es discutida por la demandada, y que dada la fecha en la cual trabajó no puede someterse al sistema jurisdiccional del Tribunal Administrativo del Trabajo de la OIT (al que posteriormente se unió la IPGRI). Respecto de la otra posibilidad, establecida en el reglamento interno de la institución: someter el caso a un comité de apelaciones, se trata de un "mero rimedio interno", que carece de las características de una protección judicial en el sentido ya indicado, pero del cual se excluye expresamente el examen de quejas relacionadas con la expiración del contrato de trabajo del empleado (Considerato 6.6.).

Entonces, el régimen indicado es incompatible con el principio constitucional mencionado, por la exclusión del derecho de los empleados de la institución a la protección judicial ante un organismo independiente. Interpretado constitucionalmente, de acuerdo con el principio de supremacía constitucional, sobre la base de los datos normativos proporcionados por el artículo 17 del convenio ratificado con la Ley No 67/1994 (que dispone que "L'Istituto stabilirà procedure idonee per la soluzione delle controversie con il suo personale"), corresponde declarar que se prevé una obligación específica del IPGRI, para el respeto de la garantía constitucional fundamental, la creación de instrumentos de protección 
judicial ante un órgano imparcial e independiente, y de acuerdo con el mismo principio, debe estimarse que el cumplimiento de esta obligación condiciona el mismo reconocimiento de inmunidad de jurisdicción en virtud del artículo 5 del acuerdo (Considerato 6.7.). En consecuencia, el incumplimiento de la obligación en cuestión durante el periodo analizado (que excluye el recurso a cualquier forma de protección judicial de las situaciones subjetivas de los empleados de la IPGRI) ha impedido la operatividad de la norma que otorga la inmunidad, de modo que el conocimiento de las controversias como las promovidas por Drago deben ser atribuidas al juez italiano (Considerato 6.8.).

\section{Conclusiones}

De esta breve revision podemos obtener algunas conclusiones:

(a) En el caso de las sentencias francesas, estas son más breves y directas, fundamentandose además en las convenciones internacionales y otras razones de derecho internacional, en una doctrina de derecho internacional ampliamente desarrollada, que vincula el quid pro quo (la obligación de las or de otorgar vías de solucion de conflictos) la concepción francesa de orden público internacional. Otro aspecto interesante es que hay sentencias que presentan un enfoque material y no solo formal respecto de la concesion de vias alternativas de solucion de conflictos por parte de las OOII, que exige una resolución conforme a un procedimiento que ofrezca garantías de un juicio justo en el sentido del artículo 6. 1. del CEDH

(b) En el caso de las sentencias italianas, el camino es diferente pero sus resultados podrían llegar a ser similares. Por ejemplo, la mención a la Convención de la ONU sobre las Prerrogativas e Inmunidades de los Organismos Especializados, de la que derivaría la obligación de quid pro quo, o la doctrina de la sentencia del TEDH sobre el caso Waite \& Kennedy c. Germany. Otro extremo interesante es un fundamento constitucional (derecho interno), donde era constitucionalmente legítima la inmunidad otorgada a una OI cuando la convención que eximía ciertos conflictos de la jurisdicción italiana garantizaba la protección judicial de esos litigios ante un juez imparcial e independiente, aunque elegido de acuerdo con procedimientos y criterios diferentes de los que se encuentran en el ordenamiento jurídico interno.

(c) En ambos países, las sentencias mencionadas efectúan una evaluación o ponderación de valores o intereses en juego relevantes al 
caso. Es decir, no se enfocan únicamente en la inmunidad de las OOII y sus fundamentos como única razón para decidir.

(d) En general, las sentencias citadas consideran entre los elementos en juego relevantes al caso, y con el poder de participar en el balance de intereses, ciertos derechos fundamentales (particularmente, derecho de acceso a la jurisdicción) y de obligaciones internacionales en el campo de los derechos humanos.

\section{Referencias}

Berenson, William. Inmunidad de jurisdicción de organizaciones internacionales públicas en el sistema interamericano: desarrollos y preocupaciones. Organización de Estados Americanos, 2013. https://www.oas.org/es/ sla/ddi/docs/publicaciones_digital_XL_curso_derecho_internacional_2013_William_M_Berenson.pdf

Bonafe, Beatrice. "Italian Courts and the Immunity of International Organizations." International Organizations Law Review 10 (2014): 505-537.

Bonafe, Beatrice. "Italian Courts and the Immunity of International Organizations." En Immunity of International Organizations, editado por Niels M. Blokker y Nico J. Schrijve, 246-278. Lieden: Brill Nijhoff, 2015.

Consolino, Sara. "L'immunità delle organizzazioni internazionali: il caso della FAO”. Tesis de maestría, Libera Università Internazionale degli Studi Sociali, 2017. https://tesi.luiss.it/21063/1/626852_ CONSOLINO_SARA.pdf

Dominice', Christian. 'Observations sur le contentieux des organisations internationales avec des personnes prive'es". Annuaire Française de Droit International 45 (1999): 623-648. https://www.persee.fr/doc/ afdi_0066-3085_1999_num_45_1_3580

Drago, Guillaume. "Conception française de l'ordre public international". En Le Rapport annuel 2013, Composition de la Commission du Rapport et des E'tudes de la Cour de Cassation, 2013. https://www. courdecassation.fr/files/files/Publications/Rapport $\% 20$ annuel/ rapport-annuel_2013.pdf

Freedman, Rosa. "UN Immunity or Impunity? A Human Rights Based Challenge." The European Journal of International Law 25 no. 1 (2014): 239-254. https://doi.org/10.1093/ejil/cht082 
Gaillard, Emmanuel e Isabelle Pingel-Lenuzza. «International Organisations and Immunity from Jurisdiction: To Restrict or to Bypass.» International \& Comparative Law Quarterly 51 no. 1 (enero, 2002): 1-15, https://doi.org/10.1093/iclq/51.1.1

Griffth, Jhon. "Restricting the Immunity of International Organizations in Labor Disputes: Reforming an Obsolete Shibboleth." Virginia Journal of International Law 25 (1985): 1007 y ss.

Huici Sancho, Laura, El hecho internacionalmente ilícito de las organizaciones internacionales. El proyecto de la Comisión de Derecho Internacional sobre responsabilidad de las organizaciones internacionales. Barcelona: J.M. Bosch, 2007.

Kolb, Robert. Théorie du Ius Cogens International. Essai de relecture du concept. Ginebra: Graduate Institute Publications, 2001. https://books. openedition.org/iheid/4846

Nanda, Ved. «Accountability of International Organizations: Some Observations.» Denver Journal of International Law and Policy 33 no. 3 (junio, 2005): 379-390.

Novak, Gregor y August Reinisch. "Issues of Effectiveness and Legitimacy. Desirable Standards for the Design of Administrative Tribunals from the Perspective of Domestic Courts." En The Development and Effectiveness of International Administrative Law. On the Occasion of the Thirtieth Anniversary of the World Bank Administrative Tribunal, editado por Olufemi Elias, 273-302. Leiden: Martinus Nijhofff Publishers, 2012.

Reinisch, August. International Organizations before National Courts. Nueva York: Cambridge University Press, 2008.

Reinisch, August. "Comments on a Decade of Italian Case Law on the Jurisdictional Immunity of International Organizations." The Italian Yearbook of International Law 19 no. 1 (2009): 101-111. https:// doi.org/10.1163/22116133-90000122

Reinisch, August. "To What Extent Can and Should National Courts Fill the Accountability Gap?” International Organizations Law Review 10 (2013): 572-587.

Reinisch, August. "Privileges and Immunities." The Oxford Handbook of International Organizations, editado por Cogan et al. Oxford: Oxford University Press, 2016. https://doi.org/10.1093/ law/9780199672202.003.0049 
Rutsel, Martha. «International Financial Institutions and Claims of Private Parties. Immunity Obliges.» The World Bank Legal Review 3 (2012).

Ruys, Tom, Nicolas Angelet y Luca Ferro. The Cambridge Handbook of Immunities and International Law. Cambridge: Cambridge University Press, 2019. https://doi.org/10.1017/9781108283632

Schmitt, Pierre. Access to Justice and International Organizations: The Case of Individual Victims of Human Rights Violations. Reino Unido: Edward Elgar Publishing, 2017.

Schmalenbach, Kirsten. "Austrian Courts and the Immunity of International Organizations." International Organizations Law Review 10 no. 2 (2013): 446-463. https://doi.org/10.1163/15723747-01002012

Schrijver, Nico. "Beyond Srebrenica and Haiti: Exploring Alternative Remedies against the United Nations.” En Immunity of International Organizations 55, 329-341. Lieden: Brill Nijhoff, 2015.

Van Alebeek, Rosanne y Andre Nollkaemper. Privileges and Immunities of International Organizations in the Case Law of Dutch Courts." Amsterdam Center for International Law no. 2012-11 (2012). https:// ssrn.com/abstract $=2117821$

Wellens, Karel. «Fragmentation of International Law and Establishing an Accountability Regime for International Organizations: The Role of the Judiciary in Closing the Gap.» Michigan Journal of International Law (2004): 1159 y ss.

Young, Carsonn. «The Limits of International Organization Immunity: An Argument for a Restrictive Theory of Immunity Under the IOIA. Texas Law Review 95 no. 4 (2017): 889-913.

\section{Jurisprudencia}

\section{Francia}

African Development Bank c. Degboe (2005), Court of Cassation (soc.), 25 enero 2005, ILDC 778 FR 2005.

De Beaugrenier c. Unesco (2009), Court of Cassation, Chambre sociale, 11 February 2009, application No07-44240. 4 Journal de Droit International (2009) 1247-1274. https://www.legifrance.gouv.fr/ affichJuriJudi.do?oldAction $=$ rechJuriJudi\&idTexte $=J$ URITEXT 000020256970\&fastReqId $=2050500942 \&$ fastPos $=18$ 
Hintermann c. Western European Union (1997), Court of Cassation, 14 noviembre 1995, Journal du Droit International, 1997.

Illemassene c. OECD (2010), Court of Cassation (soc.), Chambre Sociale, 29 septiembre 2010, No 09-41030, ILDC 1749 (FR 2010) (France).

Mazéas c. Latin Union (2003), Court of Cassation, 30 septiembre 2003, Juris-Classeur Périodique (2004) II 10102.

M.X. c. Banque Africaine de Développement (2005), Cour de Cassation, Chambre sociale, Audience publique du 25 janvier 2005, $\mathrm{N}^{\circ}$ de pourvoi: 04-41012, Publié au bulletin. Bulletin $2005 \mathrm{~V}, \mathrm{~N}^{\circ} 16$.

Unesco c. Boulois (1998), Tribunal de grande instance de Paris, 20 octobre 1997, Rev. Arb. (1997) 575; Cour d'appel Paris (14e Ch. A), 19 juin 1998

X c. Organisation Européenne de télécommunications par satellite (EUTELSAT) (2001), Court of Cassation, 5 June 2001, Bulletin (2001) 161

\section{Italia}

Caretti c. FAO (2004), Court of Cassation s.u., 23 enero 2004, Nº 1237, AC, 2004, 1328.

Drago c. International Plant Genetic Resources Institute (IPGRI) (2007), Final appeal judgment, № 3718 (Court of Cassation, All Civil Sections); ILDC 827 (IT 2007).

FAO c. Colagrossi (1992), 18 de Mayo de 1992, Corte di cassazione, $\mathrm{N}^{\circ} 5942$, 75, Rivista di diritto internazionale, 1992, p. 407.

G.C. c. FAO (1999), Corte de Casación, sentencia del 5 de noviembre de 1999.

INPDAI C. FAO (1982), Corte di cassazione; Sezioni unite civili; sentenza 18 ottobre 1982, $\mathrm{N}^{\circ}$ 5399, Rivista di giurisprudenza costituzionale e civile, Il Foro Italiano, V. 105; Rivista di diritto internazionale, 1983.

European University Institute c. Piette, Corte di Cassazione, Judgment $\mathrm{N}^{\circ}$ 149/1999, (18 de marzo de 1999), Rivista di diritto internazionale privato e processuale, 2000, 474 y ss.

Nacci c. ICAMAS (1994), Corte Di Cassazione, judgment No 5565/1994, Rivista di diritto internacional Privato e processuale, 1995.

Pistelli c. European University Institute EUI (2005), Appeal judgment, No 20995; ILDC 297 (IT 2005), Guida al diritto 40 (3/2006), 28 October 2005; Rivista di diritto internazionale, 2006, 254 y ss. 
Rivista di Diritto Internazionale Privato e Processuale, Anno XLII, No 2 , aprilegiugno 2006, Edizioni CEDAM-Padova, 468-474, Corte di Cassazione (s.u.), sentenza 28 ottobre $2005 \mathrm{~N}^{\circ} 20995$

\section{Holanda}

Dutch Supreme Court, Mothers of Srebrenica Association et al. c. The State of the Netherlands, judgment of 13 April 2012, LJN Bw1999.

\section{TEDH}

Waite and Kennedy c. Germany (1999), Application No 26083/94, 18.02.1999, European Court of Human Rights, TEDH-ECtHR.

\section{Colombia}

Trabajadores c. Empleamos S. A., Secretaría Ejecutiva del Convenio Andrés Bello (SECAB) y la Organización de Estados Iberoamericanos (OEI), AL32952014, Corte Suprema de Justicia, Sala de Casaación Laboral (abril 9, 2014). https://www.cortesuprema.gov.co/corte/wp-content/ uploads/relatorias/la/Publicacion/AL3295-2014.pdf 